\section{Generation of Digital Surface Models in Urban Areas Using Triple-stereo Imagery}

Gl_Forum 2016, Vol.1

Page: 65 - 71

Short Paper

Corresponding Author: kerstin.kulessa@stud.abg.ac.at DOI: 10.1553/giscience2016_01_s65

\author{
Kerstin Kulessa and Stefan Lang \\ University of Salzburg, Austria
}

\begin{abstract}
Stereo or even triple-stereo satellite imagery of very high spatial resolution is a versatile source for many applications and analyses. The aim of this short paper is to demonstrate the level of accuracy that can be calculated for digital surface models using triple-stereo data in urban areas. Urban planners benefit from such results thanks to the fast processing time and low cost in comparison to other data, such as LiDAR data. In addition, the data obtained using stereo or triple-stereo technology simultaneously acquire the requisite spectral information. This study used semi-global matching (SGM), which runs a pixel-wise matching of two image pairs. SGM outputs a point cloud for each pair, which is used for the generation of digital surface models (DSMs). The result of the study is a DSM which can be used in urban areas for the estimation of urban volumes such as the vegetation volume or building cubature. Results are evaluated with respect to the overall accuracy, the number of errors, and missing values due to shadows or dark homogeneous areas.
\end{abstract}

\title{
Keywords:
}

remote sensing, triple-stereo, Pléiades, DSM, semi-global matching

\section{Introduction}

Special satellites record several images during one pass, collecting data in stereo or triplestereo acquisition mode. This opens up a range of applications, of which some focus on urban areas, for example for the 3D modelling of construction sites (Carl et al., 2013). Urban areas are rapidly changing and expanding environments. This is a challenge for regional development and particularily urban planners, who need regularily updated data on the city to maintain urban living standards ('quality of life') and improve urban planning, notably in relation to green spaces, which are perceived differently by citizens (Carrus et al., 2015). Two-dimensional maps and orthophotos used to form the planning basis of their work. Nowadays, with the increasing availability of 3D technology and 3D city models, urban features and height characteristics can be more realistically represented. But these representations need to be fed with recent and accurate primary data. One option is the use of LiDAR (Light Detection and Ranging) data, which allows for resolution down to the centimeter to be achieved (Gehrke et al., 2010). There are alternative options to the high 
acquisition costs and effort of dedicated flight campaigns for the calculation of height models - bi- or triple-stereo satellite imagery. The lower spatial resolution afforded by this technology is compensated for by the low cost (acquisition costs of $30-40 €$ per $\mathrm{km}^{2}$ ) and the spectral information, which is instantaneously recorded.

The objective of this study was to calculate a digital surface model (DSM) from triple-stereo satellite imagery of the Salzburg urban area and to evaluate its accuracy in terms of usability for urban areas in general. DSM-generation was carried out using the semi-global matching (SGM) algorithm, which is integrated into many software packages, for instance the ERDAS Imagine Photogrammetry Suite. The DSM generated then served as the basis for further applications and analysis in the city area, such as volume estimation of vegetation or building cubature.

\section{Methods}

\section{Study Area and Data}

The study was carried out in the conurbation of Salzburg, Austria, which is located on the northern fringe of the Alps. The study area is around $165 \mathrm{~km}^{2}$ and includes a range of topographies, such as the Gaisberg mountain in the east, and steep hills within the otherwise flat urban area. There are also a variety of structures - a mix of high and very low building density, multi-storey buildings in the inner city, and simple houses in outlying residential areas. The study area covers not only the city of Salzburg itself but also adjacent municipalities and the town of Freilassing on the German side of the Saalach river (see Figure 1). Salzburg city itself has been the focus of a series of research projects, from which multiple datasets and analyses are available for use in further analyses, comparison and validation, such as the study on urban green (Schöpfer et al., 2005).

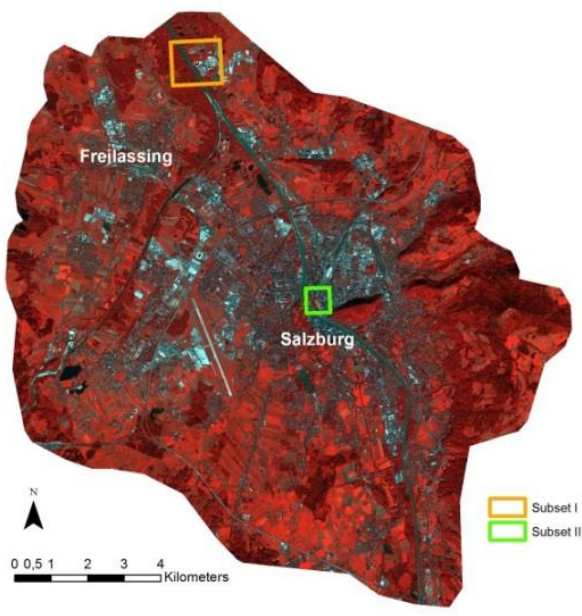

Figure 1: Pléiades-1 A multispectral image of Salzburg study area with subset locations 
For the DSM-generation, triple-stereo Pléiades-1A satellite imagery was used. The Pléiades system is composed of twin satellites in the same orbit: Pléiades-1A launched on 16 December 2011 and Pléiades-1B launched on 2 December 2012 (Astrium, 2012). The pairing of the two enables a 1-day revisit time for any location on earth. Different acquisition modes and data products can be chosen, depending on the intended usage. Options include the stereo or even triple-stereo acquisition mode for DSM generation, and 3D modelling for various applications. Eisank et al. (2015), for example, discussed the potential of Pléiadesderived DSM-generation in rough alpine terrain. Pléiades-1A and Pléiades-1B are very high resolution (VHR) satellites, with a product resolution of $0.5 \mathrm{~m}$ for the panchromatic and 2.0 $\mathrm{m}$ for multispectral (blue, green, red and near-infrared) channels. The ground sampling distance (GSD) at nadir is $0.7 \mathrm{~m}$ for the panchromatic channel, and $2.8 \mathrm{~m}$ for the multispectral channel. Pléiades-1A images were used for this study, obtained in triple-stereo acquisition mode - i.e. images were taken in forward, close-to-nadir and backward directions during one single pass (see Figure 2).

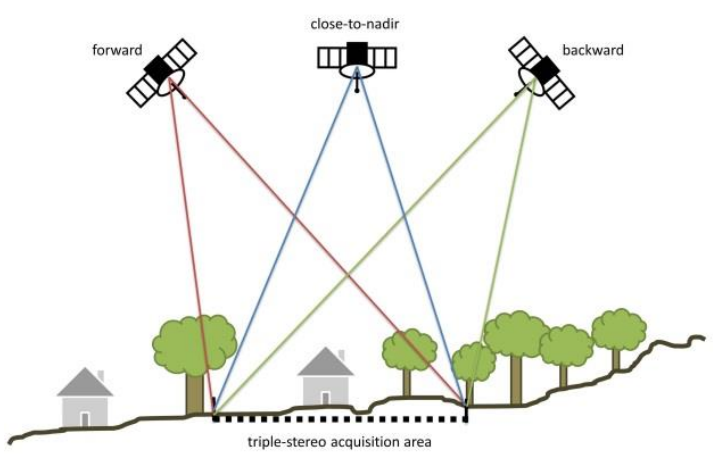

Figure 2: Triple-stereo acquisition mode (own composition)

The images were recorded on 1 September 2015 around midday local time, which results in smaller shadows in the images. The data was delivered as a primary product with a bit-depth of 12 bits; that is, the contrast of the images is very good and therefore suitable for the photogrammetry purposes intended.

The data package contained six images: three panchromatic images (forward, close-to-nadir, backward), and three multispectral images that used blue, green, red and near-infrared bands of the same locations. For the DSM-generation, only the panchromatic band was used because it has the highest spatial resolution and SGM works using only one band. Additionally, each image was linked to a Rational Polynomial Coefficient (RPC) file, which gave information about the image orientation. Table 1 shows key parameters of the three Pléiades images used for this study. 
Table 1: Parameters of Pléiades imagery (Astrium, 2012)

\begin{tabular}{|c|c|c|c|c|}
\hline $\begin{array}{l}\text { Platform/ } \\
\text { Mode }\end{array}$ & $\begin{array}{l}\text { Acquisition } \\
\text { Date }\end{array}$ & $\begin{array}{l}\text { Acquisition } \\
\text { Time }\end{array}$ & $\begin{array}{l}\text { Viewing Angle } \\
{\left[{ }^{\circ}\right] \text { along track }} \\
\text { / across track }\end{array}$ & $\begin{array}{l}\text { Ground } \\
\text { Sample } \\
\text { Distance } \\
\text { [m] }\end{array}$ \\
\hline PHR 1A/ PX & 1 Sept. 2015 & 10:17:48 UTC & $10.31 / 3.51$ & 0.72 \\
\hline PHR 1A/ PX & 1 Sept. 2015 & 10:18:02 UTC & $2.33 / 3.87$ & 0.70 \\
\hline PHR 1A/ PX & 1 Sept. 2015 & 10:18:28 UTC & $-11.66 / 4.48$ & 0.73 \\
\hline
\end{tabular}

\section{Semi-Global Matching and DSM generation}

For applications in urban areas, it is very helpful to create a 3D-model of the study area in order to obtain information on the third dimension. In this study, this was done by generating a DSM from the Pléiades-1A triple-stereo data using the Semi-Global Matching (SGM) approach (Hirschmüller, 2005).

The SGM algorithm searches the image pairs for corresponding pixels (Gehrke et al., 2010) within a defined area around a pixel. The aim is to find the pixel pairs with the highest similarity. This pixel-matching algorithm is based on cost calculation of intensity and smoothness constraints (Hirschmüller, 2008). The assumption for this algorithm is a known epipolar geometry of two or more images (Hirschmüller, 2005). The SGM approach is widely used because the results are highly accurate and the calculation quick to run (Hirschmüller, 2011). Furthermore, the SGM algorithm is embedded in various software products, hence our study used the ERDAS Imagine Photogrammetry Suite, which includes the Tridicon ${ }^{\circledR}$ Semi-Global Matching tool.

In order to use the SGM in ERDAS Imagine, we needed to create a photogrammetric project which included the stereo images used for the DSM-generation. SGM is calculated on three image pairs: front/nadir, nadir/back and front/back. The output of the stereo matching process is a point cloud for each image pair. If triple-stereo satellite imagery is used, SGM creates three point clouds based on mutual matching; for stereo data, the output would be a single point cloud. In the next step, the three point clouds are merged and error values can be filtered out. The point cloud serves as the basis for DSM-generation, which is done by rasterizing the point clouds to a defined resolution. By using the spectral information from the satellite imagery, individual urban objects like groups of trees or buildings can be extracted and their height can be determined using information from the DSM (see Figures 2a \& 2b). 


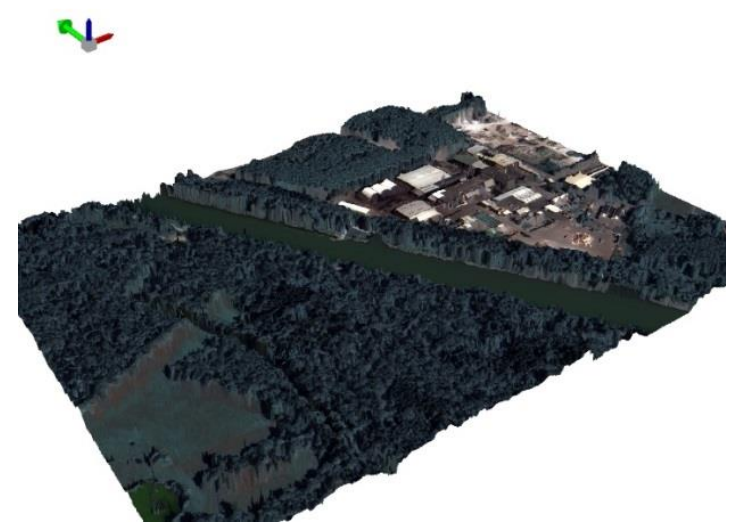

Figure 2a: Subset area I of Salzburg with height information from DSM added
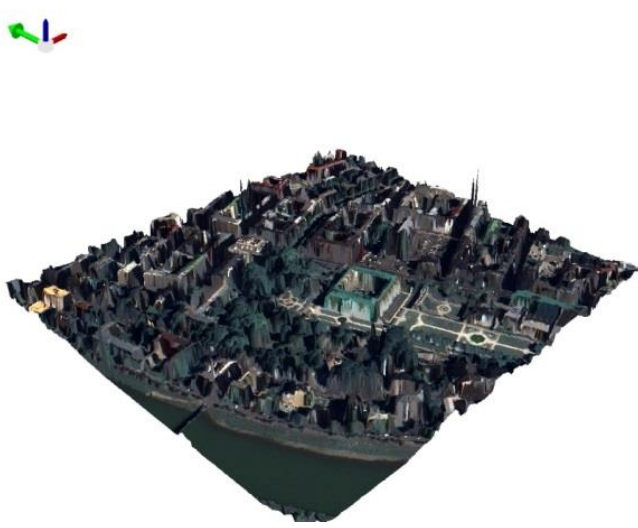

Figure 2b: Subset area II of Salzburg with height information from DSM added

This information can be used by urban planners to estimate volume relations between builtup and green areas. The volume is calculated by using the area of the object extracted from the spectral information and the height provided by the DSM.

\section{Results}

\section{Evaluation}

Extracting height information from the triple-stereo satellite data is a reasonably straightforward process. Other than for geometric and/or atmospheric corrections, preprocessing of the images is not necessary, because SGM runs even on the primary data. The point clouds can be calculated quickly: for the study area, it took about 2 hours to compute all point clouds with a $3.26 \mathrm{GHz}$ processor and $8 \mathrm{~GB}$ RAM.

The resulting point cloud was converted to a DSM raster grid; data gaps in the point cloud were interpolated. The accuracy of the DSM is dependent on the precision and correctness of the point cloud, and in order to check the accuracy, an ALS (Airborne Laserscanning) dataset from 2006 was used. This dataset covers nearly the whole study area and has a 1-m resolution. The height difference between the DSM derived from the triple-stereo satellite imagery and the ALS-dataset was calculated. In this height difference layer, dark tones or black indicate height values of the DSM, derived from triple-stereo data, that are greater than the corresponding heights in the ALS dataset; white indicates the opposite (see Figure 3). Grey areas mean little or no difference between the two datasets. 

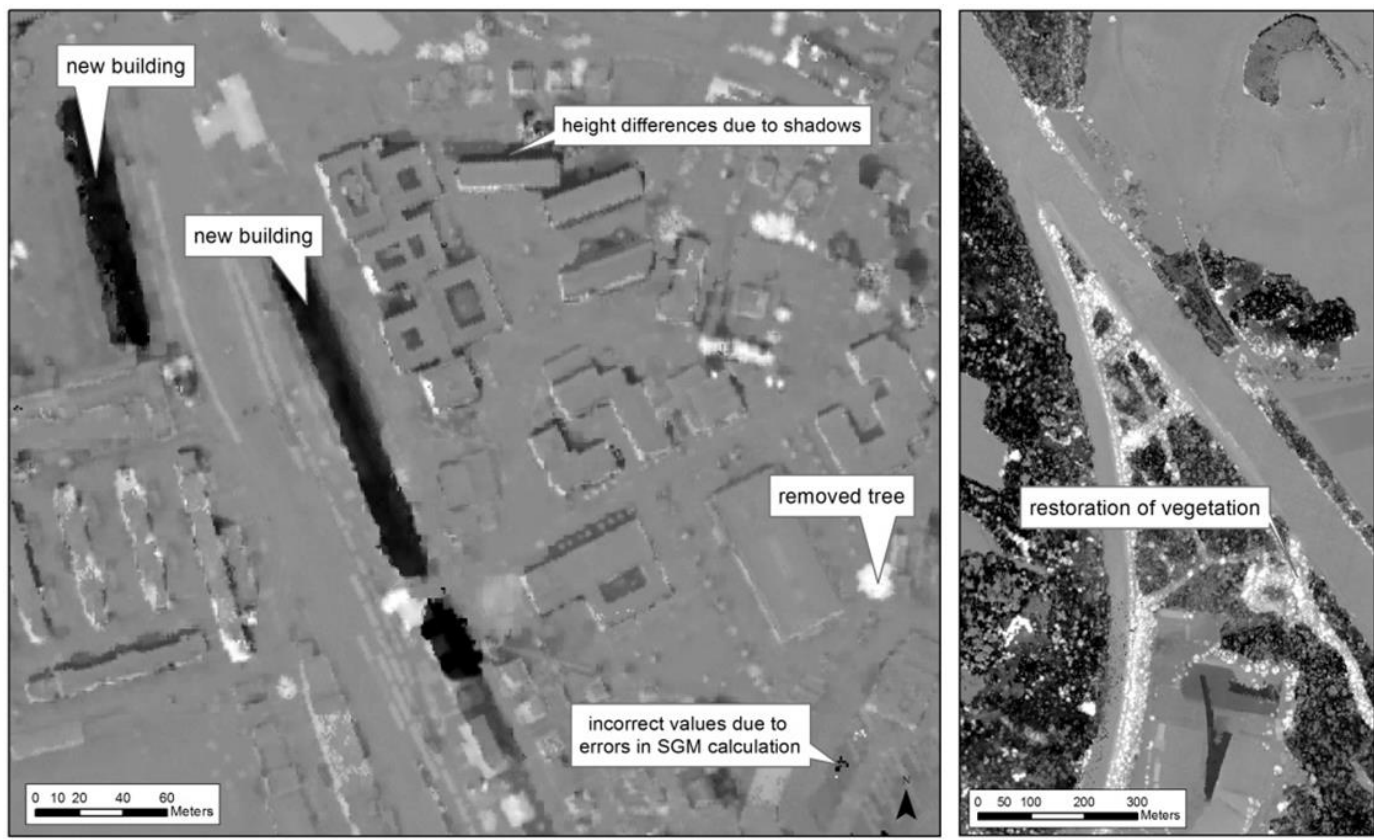

Figure 3: Height difference layer comparing heights derived from Pléiades with ALS data indicating both data inconsistencies and 'real' changes (see labels).

Large differences between the two DSMs have various explanations. There are some shadowed areas in the image, especially in the inner city of Salzburg where there is a high density of multi-storey buildings. In these areas, there are some data gaps in the point cloud where the algorithm can barely detect common pixels in the dark shadows. Some of these data gaps have to be interpolated, which causes inaccuracies. Furthermore, there are also errors attached to the matching routine itself. There is a considerable time lapse between the acquisition of the ALS data, recorded in winter 2006, and the satellite imagery from September 2015. In the meantime, there have been changes in terms of new and demolished buildings, and vegetation that has grown, been planted or been removed. For the riparian zones of the Salzach river in particular, restoration of vegetation through replanting could be identified in the difference layer (see Figure 3). Moreover, the acquisition of the ALS data was done during winter time: trees in winter show up differently from trees in leaf. The mean difference between the two height models is $0.85 \mathrm{~m}$. Triple-stereo data results in a better quality of the DSM than using simple stereo images, because the SGM algorithm calculates three point clouds for the triple-stereo data but only one point cloud for the stereo data. That is to say that SGM with three images provides a pointcloud with a higher point density for DSM calculation. Additionally, shadows in the images can be compensated for by having images from different viewing angles.

Shadows in the satellite images of the inner city of Salzburg resulting from the high density of multi-storey buildings cause errors in the matching process when pairs of similar pixels are difficult to find. This results in missing points or inaccurate values in the point cloud. Areas with missing points have to be interpolate over larger distances during the DSM generation, 
which leads to inaccuracies that have to be taken into account during subsequent analyses. The same applies to very flat, homogeneous areas.

In general, the SGM process has many advantages, because it is accurate enough to estimate volumes and heights in urban areas. Additionally, the calculation is fast and does not need particular technical skills. Furthermore, the integration of the algorithm into various software packages is advantageous, making the algorithm easier to use and readily available.

\section{Outlook}

DSM generation from triple-stereo satellite imagery has a high potential for application for various urban planning purposes. The DSM can be used in combination with the spectral information which is provided instantly by the Pléiades satellite imagery. Satellite data can be acquired regularily to perform time series analysis, maintaining comparability to carry out further analysis. Stereo and triple-stereo satellite imagery can be seen as alternatives to LiDAR, notably when the purpose justifies a $500 \mathrm{~mm}$ resolution. Processing of the satellite imagery runs smoothly, and the data needs to be acquired only once for both height and spectral information. In general, triple-stereo satellite data has a high potential for fairly rapid and cost-effective DSM-generation. There is, however, a need for further research focusing on quality criteria and a standardized point cloud generation process for this type of data in order to ensure reliable results throughout the processing chain.

\section{References}

ASTRIUM (2012), Pléiades Imagery - User Guide. Technical Reference.

Carl, S., Bärisch, S., Lang, F., D'angelo, P., Arefi, H., \& Reinartz, P. (2013) Operational Generation of High-Resolution Digital Surface Models from Commercial Tri-Stereo Satellite Data, Photogrammetric Week'13, 261-269.

Carrus, G., Scopelliti, M., Lafortezza, R., Colangelo, G., Ferrini, F., Salbitano, F., Agrimi, M., Portoghesi, L., Semenzato, P., \& Sanesi, G. (2015), 'Go greener, feel better? The positive effects of biodiversity on the well-being of individuals visiting urban and peri-urban green areas', Landscape and Urban Planning, 221-228(134).

Eisank, C., Rieg, L., Klug, C., Kleindienst, H., \& Sailer, R. (2015), Semi-Global Matching of Pléiades tri-stereo imagery to generate detailed digital topography for high-alpine regions. GI_Forum, 2015, 168-177.

Gehrke, S., Morin, K., Downey, M., Boehrer, N., \& Fuchs, T. (2010), Semi-global matching: An alternative to LiDAR for DSM generation. In Proceedings of the 2010 Canadian Geomatics Conference and Symposium of Commission I.

Hirschmüller, H. (2005), Accurate and efficient stereo processing by semi-global matching and mutual information. In Computer Vision and Pattern Recognition, 2005. CVPR 2005. IEEE Computer Society Conference on (Vol. 2, pp. 807-814). IEEE.

Hirschmüller, H. (2008), Stereo processing by semiglobal matching and mutual information. Pattern Analysis and Machine Intelligence, IEEE Transactions on, 30(2), 328-341.

Hirschmüller, H. (2011), Semi-global matching-motivation, developments and applictaions. Photogrammetric Week, Wichmann/VDE Verlag, Berlin \& Offenbach, 173-182.

Schöpfer, E., Lang, S. \& Blaschke, T. (2005), A "Green Index" incorporating remote sensing and citizen's perception of green space. International Archives of Photogrammetry, Remote Sensing and spatial information sciences, 1-6. 